\title{
MICROESTRUCTURA LEXICOGRÁFICA PARA UNIDADES FRÁSTICAS: LOS PRAGMATEMAS
}

\author{
Lexicographical Microstructure for Phrasal Groups: The Pragmatemes
}

Xavier Blanco Escoda*

\begin{abstract}
RESUMEN
Este artículo presenta los campos de microestructura que se están utilizando para la descripción de pragmatemas (enunciados fijos situacionalmente condicionados) en el diccionario PragmatLex, elaborado por el grupo de investigación $f$ LexSem (Fonética, Lexicología y Semántica) de la Universidad Autónoma de Barcelona (España) para seis lenguas: el español, el francés, el catalán, el ruso, el bielorruso y el polaco. Palabras clave: pragmatema, microestructura, diccionario multilingüe, equivalencia de traducción.
\end{abstract}

\begin{abstract}
This paper presents the microstructure fields that are being used in the description of pragmatemes (frozen statements conditioned by the situation of communication) in the dictionary PragmatLex, build by the research group $f$ LexSem (Phonetics, Lexicology and Semantics) in the Autonomous University of Barcelona (Spain). The dictionary covers six languages: Spanish, French, Catalan, Russian, Belarusian and Polish.
\end{abstract}

Key Words: pragmateme, microstructure, multilingual dictionary, translation equivalence.

Catedrático de Filología Francesa, Universitat Autónoma de Barcelona. Esta investigación ha sido subvencionada por el Proyecto FFI-2010-15229 del Ministerio de Economía y Competitividad, España.

Correo electrónico: xblancoe@gmail.com

Recepción: 9/6/2013 Aceptación: 15/11/2013. 


\section{Introducción}

Un pragmatema es un enunciado fijo situacionalmente condicionado; esto es, un enunciado cuya fijación no es de tipo semántico, sino situacional (Blanco 2010) (Mel'čuk 2012).

Ejemplos de pragmatemas podrían ser: Recién pintado; Feliz cumpleaños o Buenas noches. Algunos pragmatemas son de uso frecuentísimo y aparecen ya desde la primera etapa del aprendizaje de una lengua extranjera (Buenos días; Muchas gracias). Sin embargo, su descripción lexicográfica es casi siempre insuficiente y, en ocasiones, incluso inexistente. $\mathrm{Su}$ traducción plantea a menudo serios problemas. Así, por ejemplo, el francés Bonne nuit no recubre, en absoluto, los mismos empleos que Buenas noches.

Si bien la mayoría de los pragmatemas son poliléxicos, también encontramos un buen número de enunciados monoléxicos situacionalmente condicionados, como ¡Hola!, Empujar - Tirar [sobre una puerta] o Dígame [al teléfono].

El grupo de investigación $f$ LexSem (Fonética, Lexicología y Semántica) de la Universidad Autónoma de Barcelona está elaborando un diccionario electrónico de pragmatemas en seis lenguas: español, catalán y francés, por un lado, y ruso, bielorruso y polaco, por otro. Los pragmatemas de las seis lenguas de trabajo se están vinculando unos a otros mediante equivalencias de traducción.

La descripción y formalización de estas unidades frásticas (no se trata de unidades léxicas, aunque formalmente puedan coincidir con un lexema o con un frasema) plantea cuestiones muy diversas, pero que pueden reducirse a una sola: la definición de una microestructura adaptada a unidades frásticas situacionalmente condicionadas. Dicha microestructura deberá comportar categorías de información sui generis como, por ejemplo, las que permitan precisar la estructura conceptual subyacente al empleo del pragmatema.

En este artículo, presentaremos los campos de microestructura que estamos utilizando en estos momentos para la descripción de los pragmatemas de nuestro diccionario (al que denominamos PragmatLex). Para una presentación de estos campos (en el diccionario francés), cf. Blanco (en prensa). Por el momento, nos centraremos en las cuestiones de técnica lexicográfica, dejando para más adelante los problemas de implementación informática.

\section{Lengua del artículo}

Prag $\mu$ atLex es un diccionario multilingüe o, más exactamente, un conjunto de diccionarios electrónicos monolingües coordinados.

El primer paso consiste en especificar la lengua correspondiente al lema mediante la norma ISO 639-1 (es para el español, fr para el francés, etc.). Los códigos que nos atañen son: $b e, c a, e s, f r, p l, r u$. Un artículo del diccionario español será, pues, un conjunto estructurado y etiquetado de datos bajo la etiqueta es.

\subsection{Lema y Morfosintaxis}

A continuación, tenemos el lema. En su calidad de unidad frástica, el pragmatema plantea serios problemas de selección de la forma canónica que debe presentarse como lema. Sólo cuando la forma del pragmatema coincide con una unidad léxica y el pragmatema no tiene (o tiene muy pocas) variantes, la lematización resulta evidente.

Los pragmatemas cuya forma coincide con la de un sintagma regular en la lengua correspondiente suelen presentar también una lematización sencilla. Peligro de muerte (Nombre + Preposición + Nombre) o Servicio incluido (Nombre + Participio pasado) son lemas naturales del diccionario de pragmatemas. También lo es Buen viaje (Adjetivo + Nombre) aunque, en este caso, el lema es una reducción de Le deseo un buen viaje, forma que, junto con otras como Que tenga usted buen viaje, Haga buen viaje, etc. figurarán en la entrada como miembros del paradgima del lema (cf. 7).

La lematización puede exigir la factorización de algunos componentes del 
pragmatema, por ejemplo, de los adjetivos correspondientes al voseo y al tuteo: $A\left\{A D J_{-}\right.$ pers: $2 s\}$ salud $=A$ tu salud; $A$ su salud.

En ocasiones, el lema comporta una «casilla abierta». En Abierto de [NÚMERO] $h$. a [NÚMERO] $h$. la casilla [NÚMERO] debe prever todos los casos posibles. Notemos que, para describir correctamente, el pragmatema, no basta con permitir cualquier número en las dos posiciones, sino que es preciso disponer de una pequeña gramática local que permita controlar, por ejemplo, que en ambos casos se utiliza la forma de veinticuatro horas. También hay que excluir las secuencias en que la primera ocurrencia sea cronológicamente posterior a la primera (no es posible abrir los lunes de 20.00 h. a 16.00 h.). Para esto se requiere una potencia de cálculo equivalente, como mínimo, a una gramática de tipo 2 (o context free).

Cuando el pragmatema presenta la forma de una frase completa, acostumbramos a encontrar (al menos en el marco de una diatopía y una diacronía concretas), una forma canónica que podemos utilizar como lema: Pase sin llamar; Prohibido fijar carteles.

A veces la lematización resulta más complicada. Existen paradigmas como:

$<01>$ conservar protegido de la luz $</ 01>$

$<02>$ conservar protegido de la humedad $</ 02>$

$<03>$ conservar protegido de la luz y la humedad $<103>$

$<04>$ conservar protegido del calor $</ 04>$

$<05>$ conservar protegido del frío $</ 05>$

$<06>$ conservar en lugar seco $</ 06>$

$<07>$ conservar en lugar fresco y seco $</ 07>$

$<08>$ conservar en lugar fresco $</ 08>$

$<09>$ conservar en el frigorifico $</ 09>$ etc.

Se trata de un número importante de variantes que deberían reagruparse. Se trata de decidir qué lemas deben postularse (eventualmente, con una relación de sinonimia con otros). Una solución extrema consistiría en retener un solo lema Conservar [EN CIERTA SITUACIÓN], lo que nos permitiría abarcar también secuencias como Conservar fuera del alcance de los niños, sinónima de la más frecuente Mantener fuera del alcance de los niños. En cualquier caso, es preciso considerar cada operación de lematización de manera individual, ya que no siempre es posible proceder a los agrupamientos que, en un principio, parecen obvios. Así, por ejemplo, no resulta conveniente agrupar Consumir preferentemente antes de [FECHA / LOCATIVO] porque un pragmatema como Consumir preferentemente antes de mayo del 2012 está dando una fecha límite de utilización óptima (FLUO), mientras que un pragmatema como Consumir preferentemente antes de ver tapa (y en la tapa: mayo 2012) está indicando una fecha límite de consumo (FLC). Se trata de dos informaciones distintas, la segunda advierte un riesgo para la salud, la primera únicamente de las mejores propiedades organolépticas del producto.

\subsection{Traducción}

PragmatLex es un diccionario monolingüe coordinado (Blanco, 2001: 67). Esto significa que el contenido de los campos denominados Equivalente de traducción no debe interpretarse como una información completa en sí misma, sino como un puntero hacia una entrada del diccionario de la lengua de destino. Un pragmatema dado puede presentar sólo una equivalencia parcial con otro pragmatema. Por ejemplo, Bonne nuit es un equivalente de traducción al francés de Buenas noches, pero este último puede utilizarse perfectamente como fórmula de bienvenida (por ejemplo, bienvenida a una velada, reunión nocturna, representación teatral, concierto, etc.). En cambio, Bonne nuit, no puede utilizarse como bienvenida, deberá usarse en su lugar Bonne soirée.

Como se desprende de lo dicho antes, en la actualidad el número de campos de equivalente de traducción es de cinco para cada lengua (v.g. para el español: francés, catalán, ruso, bielorruso y polaco).

\section{Anclaje léxico, Situación y Acto de habla}

La fijación de un pragmatema se sitúa entre la estructura conceptual y la estructura 
semántica del enunciado. Esto implica que debemos tomar en consideración información de tipo extralingüístico, situacional e incluso enciclopédica.

\subsection{Anclaje léxico}

Llamamos «anclaje léxico» a la unidad léxica (puede haber más de una para cada pragmatema) que caracteriza la situación de comunicación del pragmatema. El anclaje léxico puede aparecer explícitamente o no en el enunciado del pragmatema, pero, en todo caso, siempre forma parte de su definición. Por ejemplo, incendio es un anclaje léxico evidente para No utilizar en caso de incendio. Para cierto pragmatema, lo será ascensor, pero no en todos los casos. En Prohibido aparcar, aparcar es el anclaje obvio, pero también lo es vehículo aunque no aparezca explícitamente.

\subsection{Situación}

En el campo Situación deberán figurar las informaciones siguientes (siempre y cuando sean pertinentes para la descripción de un pragmatema dado):

- las coordenadas espaciales, por ejemplo [en un letrero sobre una puerta] para Empujar;

- las coordenadas temporales, por ejemplo [el día del cumpleaños del Destinatario] para Feliz cumpleaños o [antes de empezar a comer] para Que aproveche;

- el soporte del pragmatema, por ejemplo [escrito sobre el embalaje de un producto alimenticio] para Consumir preferentemente antes de;

- cualquier otra indicación necesaria para un correcto empleo del pragmatema, por ejemplo [al contestar al teléfono] para Dígame o [a alguien que acaba de estornudar] para Jesús.

Los pragmatemas son unidades frásticas y, por tanto, estrictamente hablando no se aplican reglas gramaticales en su combinatoria. En cambio, sí obedecen a las reglas de planificación textual (incluso, a veces, son instrumentos de dicha planificación). Por ejemplo, una fórmula de despedida se dirá al final de un diálogo, no al principio ni en su transcurso. Otro ejemplo: no es pragmáticamente aceptable ordenar ¡Apunten! tras haber ordenado ¡Fuego! (salvo si debe repetirse la acción), como tampoco es pragmáticamente aceptable (salvo ironía) decir De nada si el interlocutor no ha producido antes una fórmula de agradecimiento.

\subsection{Acto de habla}

Debemos caracterizar la enunciación prototípica para cada pragmatema del diccionario. Lo hacemos en el campo Acto de habla. A fin de disponer de una pauta para etiquetar los actos de habla, utilizamos, para el español, los sintagmas propuestos en el Nivel Umbral (Slagter 1979), con ligeras adaptaciones. Algunos ejemplos serían expresión de esperanza para Buen viaje; falta de autorización para Prohibido fumar o disculparse para Lo siento mucho.

Señalemos que el acto de habla constituye el predicado central del pragmatema.

\section{Estructura semántica}

El pragmatema es un enunciado y, como tal, tiene una estructura semántica. Por ejemplo, para Buen viaje tenemos: Locutor (primer actante semántico)DESEA P (segundo actante semántico) al Destinatario (tercer actante semántico), siendo $P=q u e$ el viaje de Destinatario a Locativo con Medio de transporte sea bueno. Nótese que el hecho de hacer figurar el medio de transporte en la estructura semántica del pragmatema nos permite establecer de manera natural vínculos de sinonimia entre Buen viaje y Buen vuelo o Feliz travesía, que implican el avión o el barco.

La estructura semántica de iApunten! sería Locutor (primer actante semántico) ORDENA $P$ (segundo actante semántico) al Destinatario (tercer actante semántico), siendo 
$\mathrm{P}=$ que el Destinatario apunte Arma de fuego a Locativo.

\section{Funciones léxicas}

La noción de función léxica (Mel'čuk 2007) es fundamental en formalización lingüística. Ya en la lematización, nos permiten reagrupar un gran número de secuencias que, de otro modo, quedarían dispersas. Por ejemplo, para Liquidación (venta de mercancías con rebaja de precios debida a una causa no ordinaria), tenemos: Magn (liquidación) $=$ gran $\sim, \sim$ total, Propt (liquidación) $=\sim$ por traspaso, $\sim$ por cierre, $\sim$ por reformas, $\sim$ por traslado, etc.

Mediante las funciones léxicas, se pueden factorizar también casos como Lea las instrucciones antes de (utilizar, usar, poner en marcha, poner en funcionamiento...) $N$, la formulación sería Real $(N)$, una función que nos $\mathrm{da}$, en cada caso, el valor apropiado para el verbo de utilización.

Una subclase muy importante de funciones léxicas son los intensivos y atenuativos. Ya hemos visto arriba un ejemplo de Magn. Otros ejemplos serían Magn $($ prohibido $)=$ estrictamente, categóricamente, terminantemente... Los atenuativos aplicados al acto de habla son muy importantes para el efecto ilocutorio del pragmatema: Se ruega guardar silencio frente al poco habitual Se ordena guardar silencio.

\section{Sinónimos/Antónimos y Codas}

De lo dicho arriba se desprende la necesidad de contar con una buena mesoestructura; esto es, con un sistema de remisiones internas que vinculen entre sí las entradas del diccionario. Un elemento clave es la indicación de sinónimos y antónimos.

El sinónimo puede ser bastante aproximado (Consumir antes de y Fecha de caducidad; Usted no tiene la prioridad y Ceda el paso) o bien puede tratarse de un parasinónimo (sinonimia parcial): Chinchín es un sinónimo parcial de $A$ la salud de $N$, pero no permite la expresión del actante *Chinchín por N. Buen vuelo es un sinónimo de mayor intensión semántica que Buen viaje.

El sinónimo también puede estar marcado desde el punto de vista diasistemático, así por ejemplo, decir Salud o Jesús a alguien que acaba de estornudar no cambia nada al acto de habla, pero caracteriza al locutor de distinto modo.

Es interesante constatar que muchos pragmatemas presentan sinónimos intersemióticos. Peligro de muerte tiene como sinónimo intersemiótico un triángulo de peligro con una calavera; en cambio, Peligro de muerte por electrocución tiene como variante un triángulo de peligro con una silueta humana cayendo fulminada por un rayo. También podemos tener una señal sonora o luminosa como sinónimo intersemiótico de un pragmatema. PragmatLex acoge, pues, ficheros de imagen y de sonido.

En cuanto a los antónimos, nos permiten dar cuenta de ciertas series, en especial de pares de pragmatemas vinculados por la antonimia; un ejemplo obvio sería el par Tire/Empuje (o, por supuesto, sus variantes diatópicas: Hale/ Empuje, etc.).

También daremos cuenta de determinadas extensiones de los pragmatemas que, sin un gran aporte semántico, completan rítmicamente al lema: Liquidación total. Precios increíbles o Precios de escándalo. Denominamos coda a estas extensiones.

No hay que confundir la coda con la réplica a un pragmatema (Gracias / De nada). Notemos que muchas réplicas están diacrónicamente marcadas: Bienvenido / Bien hallado, que ya prácticamente no se usa. Tampoco hay que confundir la coda con una sucesión de pragmatemas de significados diferenciados que obedecen a una planificación textual, por ejemplo: Este anuncio es de un medicamento. Lea detenidamente las instrucciones de uso. En caso de duda consulte a su farmacéutico.

\section{Paradigma}

Los pragmatemas no tienen flexión como tal (aunque a menudo incluyan elementos con 
flexión), pero puede aparecer, como hemos visto, bajo una considerable variedad de formas. Debemos inventoriar estas formas en el diccionario, a fin de cubrir todas las ocurrencias de un pragmatema dado y poder observar fenómenos de discurso (como, por ejemplo, la forma más frecuente de un pragmatema) Ahora bien, dichas formas no pueden preverse mediante paradigmas de flexión, ya que cada pragmatema presenta un paradigma idiosincrático. Ya hemos visto un ejemplo de paradigma con diversas casillas semi-abiertas sería: Abierto / Abierto de lunes a viernes / Abierto de 9 h. a 15.00 h. de lunes a viernes / Abierto de lunes a viernes, miércoles tarde cerrado, etc.

\section{Conclusión}

Como conclusión, nada mejor que resumir los campos básicos de una entrada estándar de PragmatLex: un lema, una clase morfosintáctica, una serie de equivalentes de traducción (en las lenguas del proyecto), un anclaje léxico (que da, al mismo tiempo, elementos fundamentales de la definición del pragmatema), la indicación del acto de habla, una estructura semántica (representada en lógica de predicados), una serie de funciones léxicas (según el modelo ya citado), una serie de sinónimos y antónimos, una coda (cuando el pragmatema la presente) y un paradigma que ofrezca el mayor número posible de realizaciones en discurso del pragmatema.

Quedarían otros campos, como el correspodiente a la definición, el ámbito de especialidad o las réplicas (para pragmatemas con estructura binaria). Tampoco hemos discutido el engarce con el diccionario de los campos de contenido icónico o sonoro. Iremos precisando el contenido y la forma de estos campos a medida que el número de entradas de PragmatLex nos lo permita.

\section{Bibliografía}

Blanco, Xavier. (2001). "Dictionnaires électroniques et traduction automatique espagnol-français". En Langages 143: 49-70.

Blanco, Xavier. (2010). “Los frasemas composicionales pragmáticos". En Mogorrón, Mejri (eds): Opacité, Idiomaticité, Traduction, Universidad de Alicante: 19-32.

Blanco, Xavier. [en prensa]. "Microstructure évolutive pour un dictionnaire de pragmatèmes". En Actes des 9èmes Journées Scientifiques LTT. Université Paris 13.

Mel'čuk, Igor. (2007). "Lexical Functions". En Burger et al. (eds): Phraseologie, Phraseology, Walter de Gruyter: 119131.

Mel'čuk, Igor. (2012). "Semantics: From Meaning to Text". John Benjamins Publishing Company: 119-131.

Slagter, Peter J. (1979). Un Nivel Umbral. Estrasburgo: Publicaciones del Consejo de Europa. 